Rev. salud pública. 14 sup (1): 32-42, 2012

\title{
Condiciones de salud y trabajo de las mujeres en la economía informal Bogotá 2007
}

Women in Informal economy health and work conditions Bogotá. 2007

\author{
Nidia R. Sotelo-Suárez ${ }^{1}$, Jorge L. Quiroz-Arcentáles ${ }^{1}$, Charo P. Mahecha- \\ Montilla² y Paola A. López-Sánchez ${ }^{2}$
}

1 Dirección de Salud Pública, Secretaría Distrital de Salud de Bogotá. Colombia. NRsotelo@saludcapital. gov.co, leoquiroz45@hotmail.com.

2 Estudiantes de la Especialización en Salud Ocupacional de la Universidad Manuela Beltrán, Bogotá, Colombia. charopmm@hotmail.com, palsandrea@hotmail.com

Recibido 30 octubre 2009 /Enviado para Modificaciones 15 Marzo 2010/Aceptado 3 Mayo 2010

\section{RESUMEN}

Objetivo Determinar las condiciones de trabajo y salud de las mujeres que laboran en la informalidad en Bogotá y recomendar líneas de acción prioritarias para prevenir las enfermedades y accidentes relacionados con el trabajo y sus secuelas.

Metodología Estudio de tipo observacional descriptivo, utilizó como base la información recolectada por los equipos locales del ámbito laboral durante el 2007 en las intervenciones que en el marco del Plan de Atención Básica. En total se incluyeron en el estudio 3936 mujeres trabajadoras en la economía informal.

Resultados El estudio reveló condiciones de precariedad de las mujeres en este sector de la economía. Se reflejan de manera precisa bajo nivel educativo, ingresos por debajo del salario mínimo legal, exposición a condiciones de trabajo deteriorantes para la salud, con largas jornadas laborales que generaron una baja disponibilidad de tiempo para el goce de actividades de ocio y tiempo libre. Adicionalmente, el $75 \%$ de las mujeres incluidas en el estudio son cabeza de familia, lo cual las predispone a una mayor carga familiar y social.

Conclusiones Se sugiere una mayor atención al fenómeno de la informalidad para evitar la condición de desprotección de las trabajadoras. Como una estrategia puntual se propone una mayor articulación entre la academia y las instituciones responsables del desarrollo de políticas públicas que permitan conocer a fondo fenómenos recurrentes en la población estudiada y así implementar programas que promuevan mejores condiciones de calidad de vida.

Palabras Clave: Condiciones de trabajo, trabajo de mujeres, riesgos laborales, (fuente: DeCS, BIREME). 


\section{ABSTRACT}

Objective: To determine work and health conditions of women working in informal economy in Bogotá, as well as to recommend priority action plans for preventing illnesses and accidents related to work and their consequences.

Methodology: Descriptive observational study. This study analyzed information collected by local groups in work-related contexts during 2007 as part of a set of forms of intervention established according to the Plan de AtenciónBásica (Primary Care Plan). This plan included 3715 units of informal work from every point of the city. 3936 women registers in all were analyzed.

Results: This study reveals evident precariousness conditions of women in this economic sector. Specifically, there are certain evident conditions that had shown in this study such as low education levels, incomes below the minimum legal standards, high exposure to damaging health work conditions, long work hours, and few hours for leisure and free time. Furthermore, $75 \%$ of women included in this study are female head of household. This condition makes them more vulnerable to a heavier family and social burden.

Conclusions: Closer attention to informal economy phenomenon is suggested in order to avoid vulnerable conditions of working women. Besides, it is necessary to improve the articulation between Academy and forms of intervention through public policies for knowing deeply repeated phenomena regarding the population of study. This articulation can be thought for implementing programs designed for improving women's quality of life.

Key Words: Work,occupational accidents, gender, health, working women working conditions (Source: MeSH, NLM)

$\mathrm{E}$ n Colombia la proporción de subempleo por insuficiencia de horas y bajos salarios pasó del $34 \%$ para el 2006 al 33,1 \% en el 2008 y para Bogotá del 33,8 \% en el 2005 al 30,7 \% en el 2008 (1). La tasa de desempleo en Colombia pasó entre el 2004 y el 2008 de 12,5 \% a 11 \% lo que representa un descenso del 1,5\%. Bogotá en el 2005 presentaba una tasa de desempleo del 12,8 \%, y pasó a una tasa de 10,1 \% en el 2008. Esto significa que en Bogotá, las mujeres son las más afectadas por el desempleo con una tasa 1,5 veces más alta que la de los hombres.

El incremento en el porcentaje de trabajadores y trabajadoras en la Economía Informal en el país se presenta en ambos sexos, siendo más representativo en las mujeres. Esta diferencia podría explicarse por la mayor tasa de desempleo en las mujeres y por la desigual capacidad de absorción de la mano de obra femenina en nuestra economía, además de considerarse que este sector permite a las mujeres desempeñar de manera paralela sus actividades domésticas, reproductivas y de servicio. Las ramas 
de la actividad económica informal en que se insertan las mujeres en mayor proporción que los hombres son el comercio y los servicios comunales sociales y personales, las ramas en las cuales la proporción es similar son los servicios financieros y la industria manufacturera, en las demás, la proporción de hombres es mayor que la de las mujeres.

En Colombia la tasa global de ocupación en el 2005 era del 51,6 \% siendo para los hombres de 65,5\% mientras que para las mujeres era del 39,1 \% en ese mismo año. En Bogotá la tasa de ocupación para el 2005 era del 55,1 \%, (63,5 \% en los hombres y 48,1 \% en las mujeres) (2). Situación que puede explicarse por la responsabilidad que históricamente se les ha dado a las mujeres sobre el cuidado de la casa, de los hijos, de los enfermos y de la realización de actividades voluntarias sociales.

En un análisis realizado en 1997 por la OPS, se determinó que los empleados formales ganaban en promedio 469447 pesos más que los empleados informales (Los hombres formales ganan en promedio 478172 pesos más que los hombres informales y las mujeres formales ganan 572056 pesos más que las mujeres informales). Evidenciándose una importante brecha entre los sexos no sólo entre sector formal e informal, sino también al interior del mismo sector económico (3).

\section{METODOLOGÍA}

Se desarrolló un estudio observacional descriptivo para determinar las condiciones de vida, salud y trabajo de las mujeres que laboraban en la economía informal en Bogotá en el año 2007. Se aplicó una encuesta de caracterización a las trabajadoras de la economía informal beneficiarias del programa salud al trabajo desarrollado por las 14 Empresas Sociales del Estado en las veinte localidades del Distrito en el marco del Plan de Intervenciones Colectivas en el 2007. La encuesta de caracterización de condiciones de salud y trabajo fue construida y validada por un grupode expertos en temas de género, enfoque diferencial y salud y trabajo de la Dirección de Salud Pública de la Secretaría Distrital de Salud.

La población de estudio la constituyeron las mujeres que laboraban como asalariadas en las unidades de trabajo informal incluidas en el programa salud al trabajoy las mujeres que trabajaban por cuenta propia en las actividades económicas prioritarias de cada localidad. Los criterios 
para la priorización de las actividades económicas fueron la densidad de unidades de trabajo de una misma actividad en la localidad, y que dicha unidad fuese calificada como informal, teniendo en cuenta aspectos tales como el tamaño de la empresa, el cumplimiento normativo y la afiliación de las trabajadores y trabajadoras al Sistema General de Seguridad Social. El término Unidad de Trabajo Informal se aplicóa aquellas pequeñas unidades productivas o de servicios de trabajadores y trabajadoras en la Economía Informal que constituyen una unidad de operación para el programa salud al trabajo.

Se hizo un muestreo por conveniencia teniendo en cuenta que la participación de las unidades de trabajo informal y las trabajadoras fue voluntaria. Por tanto los hallazgos y conclusiones solo son aplicables a este grupo poblacional. Se encuestaron 3936 mujeres trabajadoras en la Economía Informal

\section{RESULTADOS}

Actividades económicas en las que participan las mujeres evaluadas

Se evidenció que la informalidad está presente de manera importante en las veinte localidades del Distrito y que las mujeres participan en procesos productivos diversos, concentrando su actividad en los sectores de servicios, comercio y manufacturero (Figura 1). En total se identificaron 33 actividades económicas. Hay que tener en cuenta que la selección fue realizada por los profesionales del programa en cada localidad con base en la vocación productiva de la localidad y la definición de las actividades económicas prioritarias de acuerdo a la exposición de los trabajadores y las trabajadoras a condiciones de trabajo deteriorantes, por lo que las actividades incluidas en la muestra pueden no reflejar necesariamente la participación de las mujeres en el sector informal en su conjunto. El factor de riesgo más frecuentemente identificado en las empresas que ocupan a las trabajadoras evaluadas fue la carga física, seguido del riesgo físico, el biológico, el mecánico y el Psicosocial. En el sector formal aparece como el factor de riesgo más prevalente la carga física, seguida de los factores de riesgo psicosocial, los químicos y los físicos. 
Figura 1. Distribución proporcional de las trabajadoras intervenidas según actividad económica.

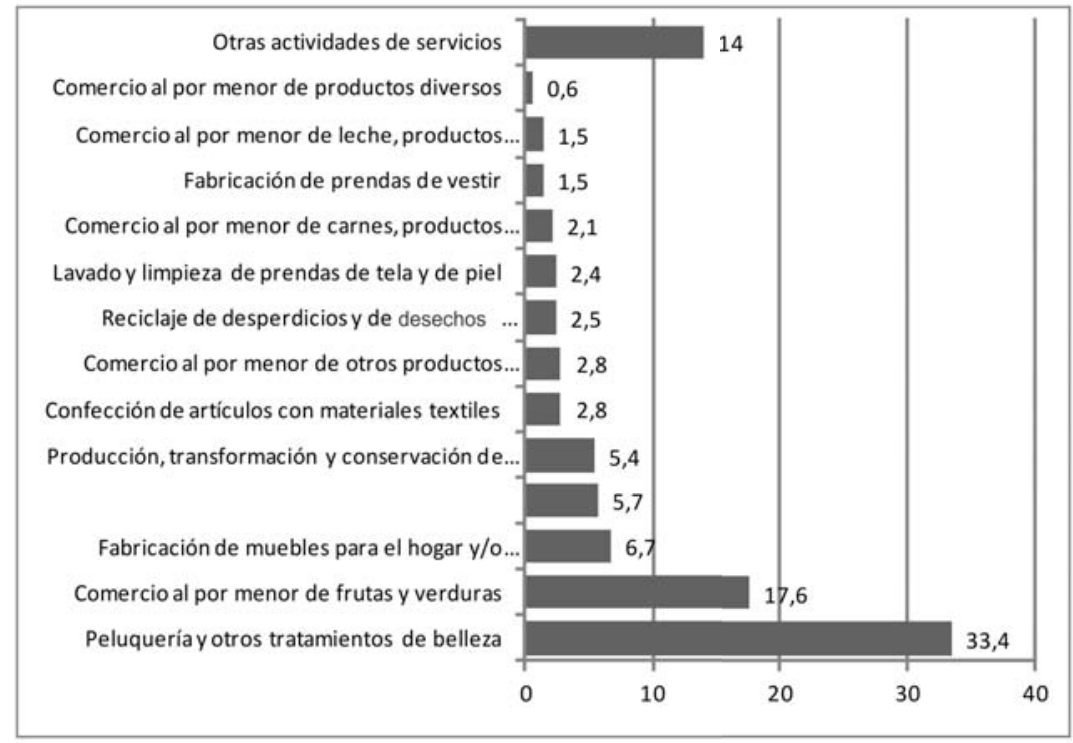

Fuente: Base de datos del ámbito laboral, SDS Bogotá 2007.

Otra característica que se hizo evidente es que las mujeres del grupo evaluado se vinculan en los procesos productivos desde la adolescencia y que pueden permanecer en ellos hasta edades avanzadas, esto puede deberse a fenómenos como la baja inserción y/o permanencia en el sistema educativo, el embarazo adolescente, la mínima capacidad del mercado laboral para ofrecer nuevos empleos de calidad o la existencia de mujeres mayores que no tienen protección social por lo que deben realizar actividades de subsistencia. En futuras investigaciones deben explorarse estas asociaciones junto con el estudio de la carga económica de las mujeres cabeza de familia que tienen una triple carga porque además de trabajar deben atender las necesidades de su familia, las propias de la reproducción y el cuidado de terceros entre los cuales se identifican personas en condición de discapacidad. Esto determina patrones de desgaste característicos de los esfuerzos que deben realizar para cumplir con su función social.

Condiciones socio económicas de las mujeres evaluadas

Tal como se evidencia en el Figura 2, el 54,2 \% de las mujeres evaluadas tienen un nivel de formación menor que el bachillerato completo y solamente 
el 13,9 \% tienen formación para el trabajo completa, lo cual permite plantear que cualquier propuesta para mejorar su calidad de vida, debe incluir no solo la creación de empleos de calidad, sino también la creación de condiciones que favorezcan su desarrollo personal, esto pone sobre el tapete la discusión sobre qué criterios componen la categoría de trabajo digno para las mujeres, que para algunos incluye la posibilidad de conciliar las labores productivas con el cumplimiento del plan de vida personal.

Figura 2. Distribución de las trabajadoras según nivel educativo

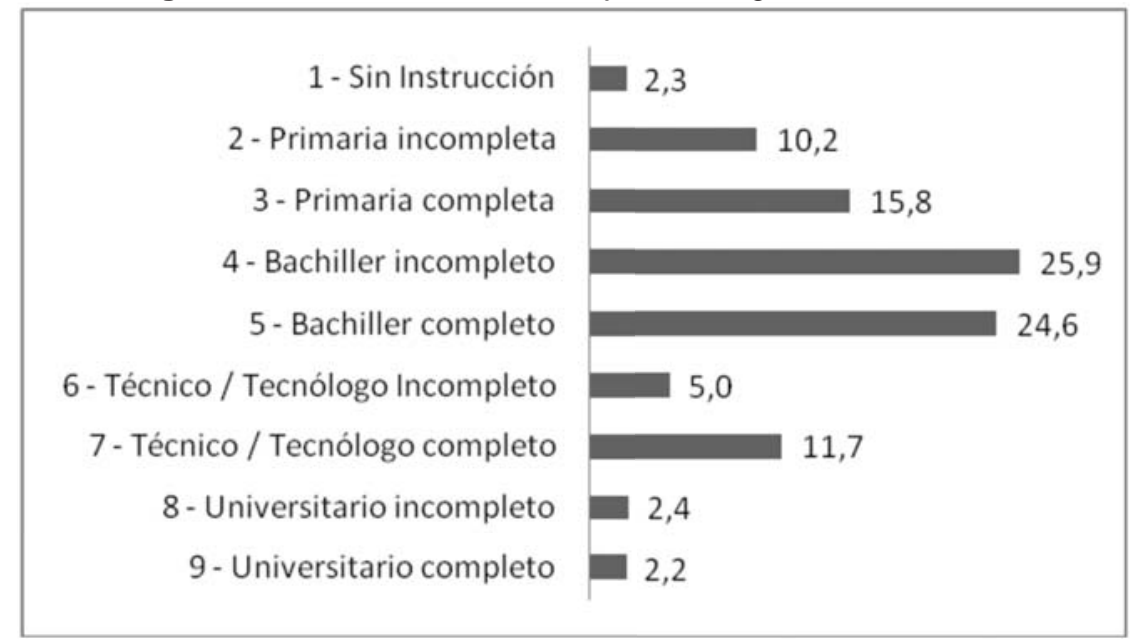

Fuente: Base de datos del ámbito laboral, SDS Bogotá 2007.

Por otra parte, el hecho de que mujeres con formación de nivel tecnológico o universitario estén vinculadas a la economía informal plantea la necesidad de explorar las causas de este fenómeno que puede estar asociado a una decisión personal de formar parte de la Economía Informal, conservando el control sobre su proyecto de vida; o bien, a las dificultades estructurales del país para acceder al mercado laboral formal. Este tipo de análisis podría ser objeto de un estudio adicional.

Un 75 \% de las trabajadoras evaluadas no tienen apoyo económico de otros miembros de la familia lo que hace pensar que se trata de mujeres cabeza de familia, que en su mayoría deben mantener su hogar con 1 a 2 salarios mínimos, lo que favorece la reproducción de una sociedad profundamente inequitativa. Esto es más evidente si se analiza la situación del 19 \% de las mujeres evaluadas que ganan menos de un salario mínimo (Figura 3) 
Figura 3. Proporción de trabajadoras según nivel de ingreso individual.

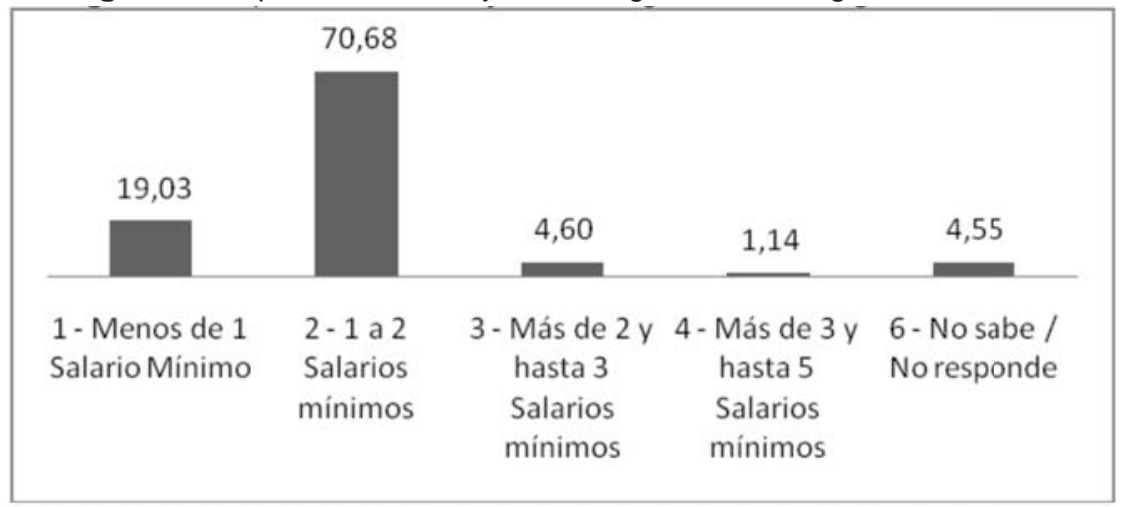

Fuente: Base de datos del ámbito laboral, SDS Bogotá 2007

La mayoría de las mujeres evaluadas habitan en estratos uno y dos, lo que puede estar determinado por la baja capacidad de consumo dado sus ingresos. También se encontró que el 37 \% de las mujeres evaluadas habitan viviendas de estrato 3 . El pequeño porcentaje que vive en estrato 4, puede pertenecer a la población de trabajadores en la economía informal por escape o decisión de acuerdo a las definición del Banco Mundial en el 2007 y hace referencia a aquellos trabajadores o trabajadoras que no pertenecen a la economía formal porque no les interesan los costos y/o trámites para acceder a la misma.

Condiciones de empleo de las mujeres evaluadas

Se hace evidente la violación de los derechos de las trabajadoras, ya que el $75 \%$ no tiene un contrato de trabajo y el $12 \%$ trabaja por obra o labor contratada.Esto hace evidente las precarias condiciones en que trabajan las mujeres evaluadas; sin primas, cesantías, vacaciones, horas extras, licencia de maternidad, ni afiliación a la seguridad social. Adicionalmente, el 69.6\% tenían como forma de pago el destajo, lo que pone en evidencia una mayor vulnerabilidad dado que este tipo de trabajo se ha asociado con la omisión de las normas de prevención con el fin de obtener mayores ingresos lo que incluye jornadas más largas y más días de trabajo a la semana. (Figura 4).

El 81 \% de las mujeres refiere estar afiliada a alguno de los regímenes de seguridad social en salud, lo cual corresponde a la evolución de la 
cobertura de la atención en salud, sin embargo solo el 48 \% pertenece al régimen contributivo. $\mathrm{Al}$ analizar si las afiliados al régimen contributivo son las cotizantes se encontró que un $11 \%$ son beneficiarias. Todo lo anterior puede indicar que los eventos en salud relacionados con el trabajo son atendidos en un $63 \%$ con cargo a las EPS subsidiadas o por el régimen vinculado. Del total de las mujeres encuestadas solo el 6,4 \%, o sea 253, se encuentran afiliadas al régimen de pensiones y a riesgos profesionales lo que ratifica lo mencionado en el párrafo anterior.

Figura 4. Distribución de mujeres según forma de pago

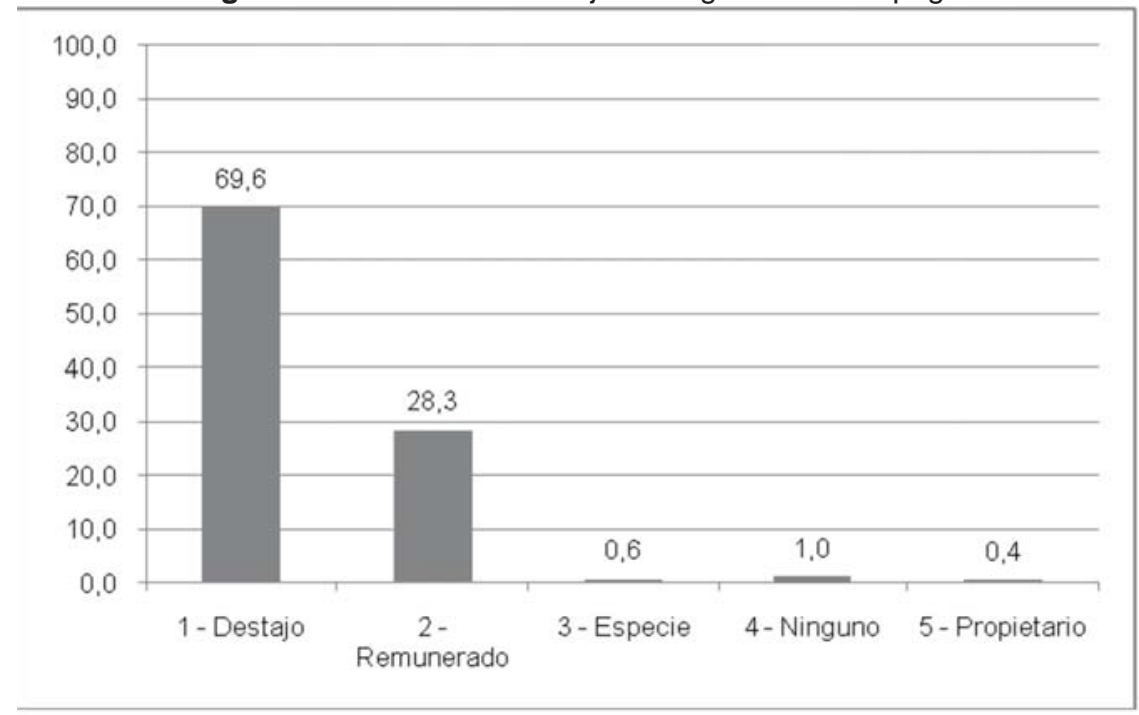

Fuente: Base de datos del ámbito laboral, SDS Bogotá 2007

Condiciones de salud de las mujeres evaluadas

En el grupo evaluado se encontró la mayor prevalencia de enfermedad relacionada con el trabajo en la actividad económica de peluquería y otros tratamientos de belleza, le siguen en frecuencia el Comercio al por menor de frutas y verduras, el Expendio a la mesa de comidas preparadas en restaurantes, y la Fabricación de muebles para el hogar y/o oficina. Se anota que la prevalencia calculada por este método no es comparable con la que se obtiene en el Sistema General de Riesgos Profesionales (SGRP), que es el resultado de un proceso de calificación, pero que a su vez se ve afectado por el subregistro. 
El $15 \%$ de las mujeres refirieron haber sufrido alguna enfermedad relacionada con el trabajo, el sistema más frecuentemente afectado fue el osteomuscular con una prevalencia de 7,6 \% seguido del sistema respiratorio y el visual. En el SGRP las primeras causas de enfermedad profesional son las del sistema osteomuscular seguida de las enfermedades de origen respiratorio, patología auditiva y dermatitis, esto indica que los hallazgos encontrados en la muestra son consistentes para los dos grupos de enfermedades profesionales que se presentan con mayor frecuencia en el país.

Varios hechos se hicieron evidentes al analizar las características de las mujeres que reportaron enfermedad relacionada con el trabajo, en primer lugar, se encontró que el sistema se seguridad social en salud cubre la atención de las patologías relacionadas con el trabajo en el 63 \% de los casos, en segundo lugar se determinó que las más afectadas son las mujeres con bajos ingresos y bajo nivel educativo lo que aumenta la vulnerabilidad ante una contingencia de salud. En tercer lugar se evidenció que las mujeres que tenían jornadas superiores a las 8 horas eran las más afectadas, lo cual puede estar relacionado con un mayor tiempo de exposición a condiciones de trabajo nocivas. Es importante aclarar que dicha jornada laboral no incluye las horas adicionales que disponen las mujeres para el desarrollo de las actividades domésticas, reproductivas y sociales.

El 11,2 \% de las mujeres evaluadas refirieron haber sufrido un accidente relacionado con su trabajo en el último año. La actividad económica con mayor frecuencia de presentación de accidentes de trabajo fue la fabricación de productos metálicos con una proporción de $63 \%$, seguida de la fabricación de calzado de cuero, piel, materiales textiles, caucho y plástico con una proporción del 32,6 \% y del expendio a la mesa de comidas preparadas en restaurantes con una prevalencia del 31,4 \%. En cuanto al número de horas de trabajo al día y la presentación de accidentes relacionados en el trabajo, la mayor proporción se encuentra en el grupo de mujeres que trabajan entre 9 y 12 horas diarias con una tasa del $12 \%$, seguido del grupo que trabaja entre 4 y 8 horas con un 11,2\%. Las partes del cuerpo más afectadas con los accidentes relacionados con el trabajo fueron las manos y dedos, seguido de los brazos, los miembros inferiores y la espalda. 


\section{DISCUSIÓN}

Al igual que en la economía formal, las enfermedades osteomusculares y del sistema respiratorio, aparecen como las prioritarias para el diseño de programas de prevención y atención temprana y oportuna, también se encontró que los factores de riesgo que deben priorizarse para las intervenciones son la carga física, los factores de riesgo físico, los biológicos y los psicosociales (7). Los accidentes relacionados con el trabajo en las mujeres que trabajan en la informalidad, se concentran en las empresas con mayor nivel de informalidad y en las trabajadoras de menores ingresos, de menor nivel educativo y de estrato más bajo, al igual que en los trabajadores de género masculino (12).

Es evidente que existe una mayor exposición de las mujeres que laboran en la informalidad a condiciones de trabajo deteriorantes derivadas de sus condiciones de empleo (forma de vinculación, mecanismos de acceso a sistemas de protección social y nivel de ingresos), que las mismas mujeres vinculadas a la economía formal; sin contar con las actividades domésticas y reproductivas que siguen bajo la tutoría casi exclusiva de las mismas. Esta situación se hace mucho más evidente cuando se compara contra las condiciones de trabajo de los hombres que laboran tanto en la economía formal como informal (13).

\section{Recomendaciones}

Todos los actores que participan en la relación salud-trabajo deben analizar el fenómeno de la informalidad y sus consecuencias en términos de desprotección de los trabajadores y el impacto que esto tiene en el Sistema de seguridad social integral. El análisis en el Distrito debe iniciarse en el nivel local con la participación activa de los alcaldes locales. De manera particular deben plantearse mecanismos para mejorar la calidad de vida de las mujeres que participan en la economía informal.

La afiliación al SGRP, condición actual para tener acceso a los servicios de salud relacionados con el trabajo, al pago de las incapacidades, indemnizaciones y pensiones causadas por el ATEP, debe ser un tema prioritario para el análisis de la situación de los trabajadores y trabajadoras en la Economía informal. Por otro lado, la intervención de la informalidad debe contemplar acciones sobre factores determinantes y asociados como la oferta de empleo, la cobertura del sistema educativo, el embarazo adolescente y la baja cobertura del sistema de pensiones del país, por tanto cualquier propuesta para mejorar la calidad de 
vida de los trabajadores y las trabajadoras que laboran en la informalidad, debe incluir no sólo la creación de empleos de calidad, sino también la creación de condiciones que favorezcan su desarrollo personal, esto pone sobre el tapete la discusión sobre los criterios que componen la categoría de trabajo digno, que para algunos incluye la posibilidad de conciliar las labores productivas con el cumplimiento del plan de vida personal

Agradecimientos: Los autores del presente artículo, manifestamos que para el desarrollo del presente estudio, utilizamos información contenida en las bases de datos del ámbito laboral de la Secretaría Distrital de Salud, previo consentimiento de dicha institución. Esta información fue recolectada por los equipos locales de ámbito durante las intervenciones adelantadas desde la Dirección de Salud Pública en unidades de trabajo informal durante el año 2007.

\section{REFERENCIAS}

1. DANE. 2008. [Internet] Disponible en: http://www.dane.gov.co/files/investigaciones/empleo/ ech_informalidad/Itrimestre. Consultado Noviembre de 2008.

2. DANE. Encuesta Continúa de Hogares 2005.[Internet] Disponible en: http://www.dane.gov. co/files/investigaciones/empleo/ech_informalidad/Itrimestre. Consultado Noviembre de 2008.

3. Acevedo D. Mujer, trabajo y salud, tendencias e inequidades de género. Washington, DC: Organización Panamericana de la Salud; 1997.

4. Barañano M. Mujer, Trabajo, Salud. Madrid: Fundación $1^{\circ}$ de Mayo, Ed. Trotta; 1992.

5.Colombia, Departamento Nacional de Planeación: Documento CONPES Más y Mejores Empleos. Bogotá, 1995.

6. Fondo de Riesgos Profesionales. Estadística Sistema General de Riesgos Profesionales 2007. Disponible en http://www.fondoriesgosprofesionales.gov.co/Estadisticas/ ESTADISTICAS_SGRP_OCTUBRE_2007.pdf Consultada el 29-01- 2008.

7. Ministerio de la Protección Social. Informe de enfermedad profesional en Colombia 20032005. Bogotá; 2007. p. 25.

8. Organización Internacional del Trabajo. Conferencia Internacional del Trabajo. Informe VI. Seguridad Social: temas, retos y perspectivas. Capitulo IV - Igualdad de género; 2001.

9. Organización Panamericana de la Salud. Las condiciones de salud en las Américas, Edición 1990, Publicación Científica № 524. Washington, D.C.:OPS; 1990.

10. Organización Internacional del Trabajo. Convenio Sobre la Protección de la Maternidad. [1952] 2000. [Internet] Disponible en: http://www.ilo.org/ilolex/cgi-lex/convds.pl?C183

11. Roldán M. La generalización del debate sobre procesos de trabajo y reestructuración industrial en los 90. Estudios del Trabajo 1992; 3: 85-124.

12. CID Universidad Nacional y Secretaría Distrital de Salud. Análisis de la encuesta sobre condiciones de salud y trabajo en las unidades de trabajo informal con mayor grado de vulnerabilidad 2006. Universidad Nacional de Colombia. Abril de 2007.

13. Organización Internacional del Trabajo. Trabajo decente y equidad de género en América Latina. 2006. Consultado en http://www.cinterfor.org.uy/public/spanish/region/ampro/ cinterfor/newsroom/resenas/2006/tdec_gen.pdf el 27 de octubre de 2010. 